University of Nebraska - Lincoln

DigitalCommons@University of Nebraska - Lincoln

10-2008

\title{
6:1 aspect ratio silicon pillar based thermal neutron detector filled with ${ }^{10} \mathrm{~B}$
}

R. J. Nikolic

Lawrence Livermore National Laboratory, Livermore, California

A. M. Conway

Lawrence Livermore National Laboratory, Livermore, California

C. E. Reinhart

Lawrence Livermore National Laboratory, Livermore, California

R. T. Graff

Lawrence Livermore National Laboratory, Livermore, California

T. F. Wang

Lawrence Livermore National Laboratory, Livermore, California

See next page for additional authors

Follow this and additional works at: https://digitalcommons.unl.edu/chemistrycheung

Part of the Chemistry Commons

Nikolic, R. J.; Conway, A. M.; Reinhart, C. E.; Graff, R. T.; Wang, T. F.; Deo, N.; and Cheung, Barry Chin Li, "6:1 aspect ratio silicon pillar based thermal neutron detector filled with ${ }^{10} \mathrm{~B}^{\prime \prime}(2008)$. Barry Chin Li Cheung Publications. 19.

https://digitalcommons.unl.edu/chemistrycheung/19

This Article is brought to you for free and open access by the Published Research - Department of Chemistry at DigitalCommons@University of Nebraska - Lincoln. It has been accepted for inclusion in Barry Chin Li Cheung Publications by an authorized administrator of DigitalCommons@University of Nebraska - Lincoln. 


\section{Authors}

R. J. Nikolic, A. M. Conway, C. E. Reinhart, R. T. Graff, T. F. Wang, N. Deo, and Barry Chin Li Cheung 


\title{
6:1 aspect ratio silicon pillar based thermal neutron detector filled with ${ }^{10} \mathrm{~B}$
}

\author{
R. J. Nikolić, ${ }^{1, a)}$ A. M. Conway, ${ }^{1}$ C. E. Reinhardt, ${ }^{1}$ R. T. Graff, ${ }^{1}$ T. F. Wang, ${ }^{1}$ N. Deo, ${ }^{2}$ and \\ C. L. Cheung ${ }^{2}$ \\ ${ }^{1}$ Lawrence Livermore National Laboratory, Livermore, California 94550, USA \\ ${ }^{2}$ Department of Chemistry and Nebraska Centre for Materials and Nanoscience, \\ University of Nebraska-Lincoln, Lincoln, Nebraska 68588, USA
}

(Received 5 August 2008; accepted 7 August 2008; published online 29 September 2008)

\begin{abstract}
Current helium-3 tube based thermal neutron detectors have shortcomings in achieving simultaneously high efficiency and low voltage while maintaining adequate fieldability performance. By using a three-dimensional silicon $p-i-n$ diode pillar array filled with boron-10 these constraints can be overcome. The fabricated pillar structured detector reported here is composed of $2 \mu \mathrm{m}$ diameter silicon pillars with a $4 \mu \mathrm{m}$ pitch and height of $12 \mu \mathrm{m}$. A thermal neutron detection efficiency of $7.3+/-0.6 \%$ and a neutron-to-gamma discrimination of $10^{5}$ at $2 \mathrm{~V}$ reverse bias were measured for this detector. When scaled to larger aspect ratio, a high efficiency device is possible.

(C) 2008 American Institute of Physics. [DOI: 10.1063/1.2985817]
\end{abstract}

Neutron detectors are required for applications including astronomy, particle physics, and homeland security. Solid state thermal neutron detectors have advantages over the currently used helium-3 gas based technology in their inherent insensitivity to microphonics, low voltage operation, and small device footprint. This has led to the development of a variety of semiconductor based thermal neutron detectors. ${ }^{1-7}$

Moving from a gas medium to a solid state material can dramatically reduce the size of the device by increasing the density of the neutron-absorbing material. An early approach for solid state thermal neutron detection is to coat a semiconductor material with the thermal neutron absorber/converter material. Boron-10 $\left({ }^{10} \mathrm{~B}\right)$ is an attractive material for this purpose because of its high thermal neutron cross section of 3840 barns. ${ }^{8}$ In this configuration, ${ }^{10} \mathrm{~B}$ is coated onto a reverse biased semiconductor diode [Fig. 1(a)]. When thermal neutrons interact with ${ }^{10} \mathrm{~B}$, energetic ions (an alpha particle, $\alpha$, and a lithium ion, ${ }^{7} \mathrm{Li}$ ) are produced. These energetic ions will lose energy over a random track inside the detector, where their travel distances are determined by the energy range of the energetic ions. When the energy of these ions is deposited into the underlying semiconductor, electron-hole pairs are generated and subsequently collected within the diode, which shows the presence of a neutron-interaction event by the increase in current over the dark current level at a specific bias voltage. Literature results have revealed that single layers of this structure are limited to efficiencies of $\sim 4 \%{ }^{3}$ In addition, cracks and poor film adhesion due to stress built-up in the planar ${ }^{10} \mathrm{~B}$ can further limit the finite film thickness to be lower than that prescribed by energetic ion range considerations. Alternative approaches being pursued include using ${ }^{10} \mathrm{~B}$ containing compounds ${ }^{2}$ and ${ }^{6} \mathrm{LiF}$ (940 barns). ${ }^{5}$ Various designs of detector geometries to collect the generated electron-hole pairs are also being pursued. ${ }^{5}$

Here we report results on our solid state thermal neutron detector, which incorporates a three-dimensional (3D) silicon $p-i-n$ diode pillar array filled with ${ }^{10} \mathrm{~B}$ [Fig. 1(b)]. We achieved the largest volume of elemental ${ }^{10} \mathrm{~B}$ within a semi-

\footnotetext{
a)TEL.: 925-423-7389. FAX: 925-423-6394. Electronic mail: nikolic1@1lnl.gov.
}

conductor platform for neutron detection. This detector has the highest reported solid state thermal neutron detection efficiency using ${ }^{10} \mathrm{~B}$ as the converter material, without a stacking configuration, and the largest gamma to neutron discrimination for these devices reported to-date.

Our 3D pillar structured design is motivated by the contradiction in length scales for (1) the thickness for capturing a thermal neutron and (2) the travel range of the energetic neutron byproduct ions. The well known ${ }^{10} \mathrm{~B}(\mathrm{n}, \alpha)^{7} \mathrm{Li}$ reaction has a $Q$-value energy of $2.79 \mathrm{MeV}{ }^{8}$ In the ground state, which has a branching ratio of $6 \%$, the resulting $\alpha$ and ${ }^{7} \mathrm{Li}$ ions have energies of 1.78 and $1.02 \mathrm{MeV}$, respectively. In the first excited state, which has a branching ratio of $94 \%$, the resulting $\alpha$ and ${ }^{7} \mathrm{Li}$ ions have energies of 1.47 and $0.84 \mathrm{MeV}$, respectively. In addition, a gamma ray $(\gamma)$ of $476 \mathrm{keV}$ is emitted.

The design challenge can be illustrated by considering estimation of the minimum required converter material film thickness and the travel range of the resulting energetic ions. Firstly, the thickness $(t)$ of the conversion material is, to first order, dictated by the number of mean free paths $(\lambda)$ of absorbing/converting material. Where $\lambda=18 \mu \mathrm{m}$ for ${ }^{10} \mathrm{~B}$ and is related to the number density $(N)$ and microscopic cross section $(\sigma)$ through the following relation $\lambda=1 / N \sigma$. The probability of neutron interaction, $P=\exp (-t / \lambda)$, determines the required thickness of ${ }^{10} \mathrm{~B}$. A ${ }^{10} \mathrm{~B}$ film thickness of $54 \mu \mathrm{m}$

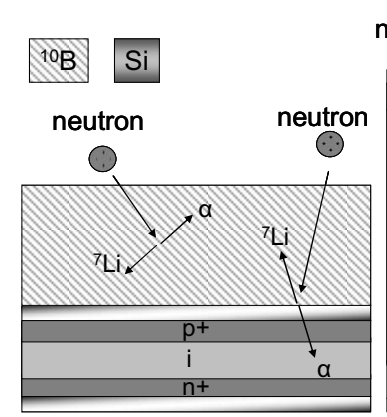

(a)

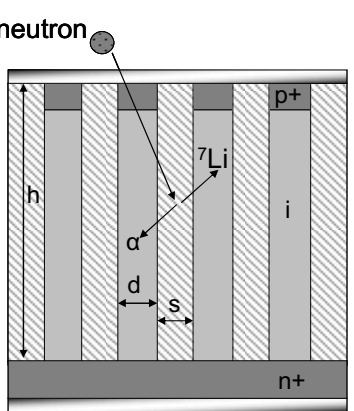

(b)
FIG. 1. Schematic of a planar (a) and pillar structured (b) solid-state thermal neutron detector. 


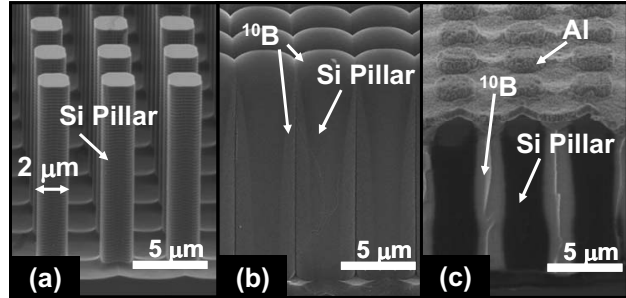

FIG. 2. Scanning electron microscopy images of $12 \mu \mathrm{m}$ silicon pillars structures: (a) as fabricated by etching, (b) after ${ }^{10} \mathrm{~B}$ deposition, and (c) after etch back of excess ${ }^{10} \mathrm{~B}$ and aluminum evaporation.

(three mean free path lengths, or $3 \lambda$ ) is needed to absorb approximately $95 \%$ of the incoming thermal neutron flux. Secondly, the travel ranges of the byproduct ions in ${ }^{10} \mathrm{~B}$ are much smaller than the required thickness for capturing a thermal neutron. Inside the ${ }^{10} \mathrm{~B}$ film, the range of alpha particles is $3.6 \mu \mathrm{m}$ and that for ${ }^{7} \mathrm{Li}$ is $1.6 \mu \mathrm{m}$, for the most favorable reaction (first excited state). It should be noted that most of the deposited energy is from the alpha particles. ${ }^{9}$ Design optimization demands simultaneously building up thicker regions of the converter material while capturing the energy from the energetic ions to achieve high efficiency for these devices.

Our pillar structured design simultaneously meets the conflicting length requirements $(\sim 3$ versus $\sim 50 \mu \mathrm{m})$ to create an optimal designer material. ${ }^{6}$ When the $3 \mathrm{D}$ pillar design is scaled in height $>50 \mu \mathrm{m}$ (increase in height is needed to accommodate the $4 \pi$ random distribution of thermal neutrons), a high efficiency device $(>50 \%)$ is predicted. ${ }^{9}$ The $3 \mathrm{D}$ pillar detector reported here [Fig. 1(b)] consists of an array of etched $\mathrm{Si} p-i-n$ pillars with $6: 1$ aspect ratio (2 $\times 2 \mu \mathrm{m}^{2}$ pillars with a $12 \mu \mathrm{m}$ etch depth or height and a separation of $2 \mu \mathrm{m}$ ) on a planar silicon substrate, arranged in a square matrix. The silicon pillars form an array of current collecting regions embedded within the ${ }^{10} \mathrm{~B}$ - the thermal neutron converter material. By etching high aspect ratio pillar structures into silicon, the result is a device that efficiently absorbs the thermal neutron flux by accommodating a large volume $(75 \%)$ of ${ }^{10} \mathrm{~B}$ within the pillar array. Additionally, the charged particles emanating from the thermal neutron ${ }^{10} \mathrm{~B}$ reaction are detected in the $p-i-n$ diodes by arranging the pillars in sufficiently close proximity.

The fabrication process began by epitaxially growing $p+$ and $i$ layers on a $n+$ silicon substrate by using an ASM Epsilon chemical vapor deposition system. The pillar diameter and spacing was then defined lithographically, followed by plasma etching to create high aspect ratio structures utilizing an $\mathrm{SF}_{6}$ approach [Fig. 2(a)]. A conformal coating of ${ }^{10} \mathrm{~B}$ was then deposited on the pillar array by chemical vapor deposition [Fig. 2(b).$^{10}$ Since the energetic ions are generated in the ${ }^{10} \mathrm{~B}$, any gap between the semiconductor and the ${ }^{10} \mathrm{~B}$ layer will decrease the detector sensitivity. Next, the "etch back" was carried out using a Plasma Quest electron cyclotron resonance etching system with a trisource gas mixture to expose the $p+$ layer for contact formation. Lastly, aluminum was sputtered onto the structures to fabricate the electrodes [Fig. 2(c)].

The electrical and radiation detection characterization described here was carried out on a detector with $2 \mu \mathrm{m}$ diameter pillars with $2 \mu \mathrm{m}$ spacing and $12 \mu \mathrm{m}$ pillar height on a test chip of $3 \times 3 \mathrm{~mm}^{2}$ (Fig. 2). Current versus voltage

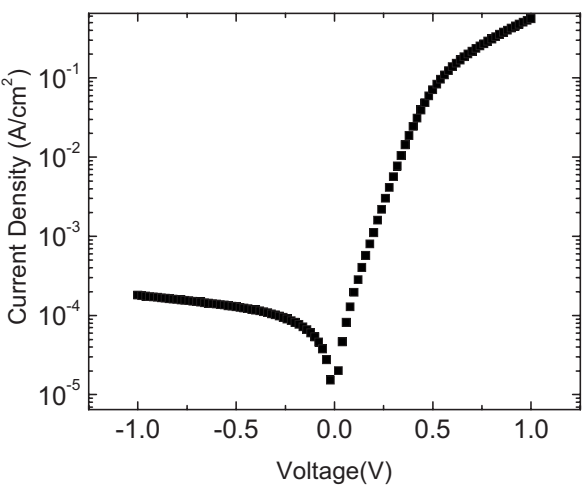

FIG. 3. Current vs voltage measurement of a $12 \mu \mathrm{m}$ tall pillar structured thermal neutron detector.

measurements illustrate a low reverse bias current density of $1.8 \times 10^{-4} \mathrm{~A} / \mathrm{cm}^{2}$ measured at $-1 \mathrm{~V}$ (Fig. 3). Low leakage current is essential for high sensitivity to neutron events because it sets the noise floor for the device. The leakage current is comprised of bulk and surface components. The reverse biased current density of an unetched planar diode structure is in the order of $10^{-7} \mathrm{~A} / \mathrm{cm}^{2}$, implying that the increase in leakage current is due to surface recombination. Further reductions can be achieved by passivating the sidewalls of the pillar array, which has a large surface area. Low voltage is also important for low power devices. The intrinsic region of the detector has a free carrier concentration of $10^{13} \mathrm{~cm}^{-3}$, which requires $-0.8 \mathrm{~V}$ for depletion.

The neutron response of this detector was measured using a ${ }^{252} \mathrm{Cf}$ fission neutron source with an intensity of $2 \times 10^{6} \mathrm{n} / \mathrm{s}$ and moderated by polyethylene blocks of $\sim 6 \mathrm{in}$. thickness to yield thermal neutrons. The measurement time was $12 \mathrm{~h}$, a $0.5 \mu \mathrm{s}$ shaping time was used and the energy was calibrated with ${ }^{243-244} \mathrm{Cm}$ source, which has an alpha energy peak of $5.5 \mathrm{MeV}$. Taking into account the size and position of the source as well as neutrons being deflected by the surroundings (concrete walls and floor) a thermal neutron intensity of $398.6+/-34.4 \mathrm{n} / \mathrm{min}$ from Monte Carlo $\mathrm{N}$ Particle ${ }^{11}$ transport simulator is obtained. The detection efficiency is then determined by integrating all counts above the noise level of $\sim 325 \mathrm{keV}$ which yields 29.1 counts/min. This in turn gives a thermal neutron detection efficiency of 7.3 $+/-0.6 \%$ at -2 V. Figure 4 shows the spectrum obtained for

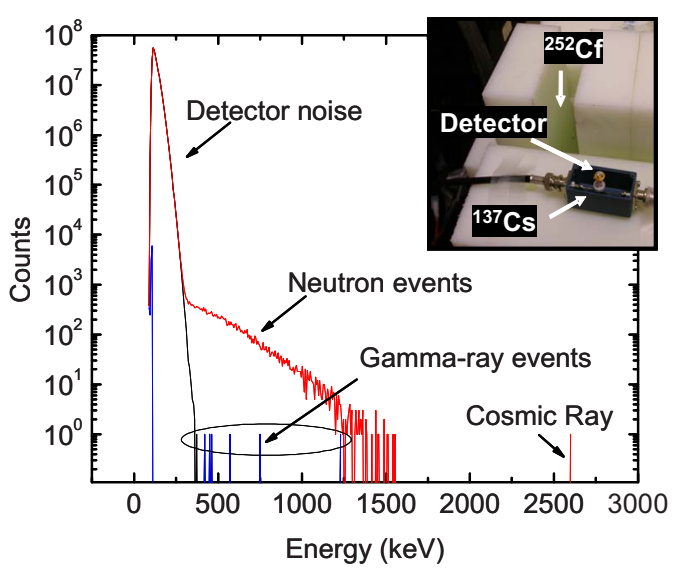

FIG. 4. (Color online) Neutron detection spectrum and gamma detection spectrum of the pillar structured thermal neutron detector with $12 \mu \mathrm{m}$ silicon pillars structures. The inset shows the test configuration. 
this measurement, and unlike for a conventional planar geometry, the energy peaks are not evident due to the random interaction profile within the 3D material matrix. Also, from Fig. 4 it is evident that the spectrum has a cutoff near 1.6 MeV. This is consistent with the full $\alpha$ energy deposition, which would give a maximum energy of $1.78 \mathrm{MeV},{ }^{8}$ (ground state). This number is not achieved due to energy loss through the material matrix. Low energy events below $325 \mathrm{keV}$, which was under the noise component, were not included in the efficiency calculation. Therefore, in order to achieve the optimal efficiency for this geometry the noise floor will need to be further decreased. The detection signal was stable with no appreciable change in integrated neutron counts when characterized between -2 and $-4 \mathrm{~V}$. The discrimination between gamma ray events and thermal neutron events was measured by exposing the detector to $662 \mathrm{keV}$ gamma rays from a $10 \mu \mathrm{Ci}{ }^{137} \mathrm{Cs}$ source, yielding six gamma ray counts during the $12 \mathrm{~h}$ measurement (Fig. 4). This is an upper bound because cosmic ray induced neutron events would also be registered. The very low measured gamma response indicates a neutron-to-gamma discrimination ratio of $\sim 10^{5}$. This result was obtained by integrating the total neutron counts (20916) divided by the total gamma counts (six) and multiplied by a normalization factor $(\sim 30)$ of the source strengths and geometries. We attribute the low gamma discrimination to the low linear attenuation coefficient $(\mu)$, which accounts for the probability of interaction per unit distance. For the energies of interest $(100 \mathrm{keV}-2 \mathrm{MeV})$ the gamma interaction cross section is dominated by Compton scattering. ${ }^{12}$ Therefore the $\mu$ can be readily determined. For the upper bound gamma energy of interest, $\gamma=2 \mathrm{MeV}$ gives a $\mu=0.10 \mathrm{~cm}^{-1},{ }^{12}$ which is dependent on the low atomic number $\mathrm{Z}=14$ and density $\rho=2.33 \mathrm{~g} / \mathrm{cm}^{3}$ for $\mathrm{Si}$. The low $\mu$, in addition to the device thickness or pillar height $(12 \mu \mathrm{m})$, are responsible for the low number of gamma counts being registered and correspondingly large neutron-to-gamma discrimination.

In summary, we have developed a device that can take advantage of the high thermal neutron cross section of ${ }^{10} \mathrm{~B}$ and accommodate a large volume fraction of this material while simultaneously addressing the conflicting length scales of neutron absorption and the resulting byproduct ranges. Radiation measurement results of our proof-of-principle device show $7.3+/-0.6 \%$ efficiency and $10^{5}$ neutron-togamma discrimination ratio with a pillar aspect ratio of $6: 1$. This is the highest efficiency reported to date for a thermal neutron detector based on ${ }^{10} \mathrm{~B}$ without a stacking configuration. It is also a step toward scaling to a depth of $>50 \mu \mathrm{m}$ for a high efficiency thermal neutron detector. The required aspect ratio for high efficiency will be on the order of 25:1 which is well within the state of the art for plasma etching techniques.

This work was performed under the auspices of the U.S. Department of Energy by Lawrence Livermore National Laboratory under Contract No. DE-AC52-07NA27344, LLNL-JRNL-405940.

\footnotetext{
${ }^{1}$ A. Rose, Nucl. Instrum. Methods 52, 166 (1967).

${ }^{2}$ K. Osberg, N. Schemm, S. Balkir, J. I. Brand, M. S. Hallbeck, P. A. Dowben, and M. W. Hoffman, IEEE Sens. J. 6, 1531 (2006).

${ }^{3}$ D. S. McGregor, R. T. Klann, H. K. Gersch, and Y. H. Yang, Nucl. Instrum. Methods Phys. Res. A 466, 126 (2001).

${ }^{4}$ D. S. McGregor, R. T. Klann, H. K. Gersch, E. Ariesanti, J. D. Sanders, and B. Van Der Elzen, IEEE Trans. Nucl. Sci. 49, 1999 (2002).

${ }^{5}$ J. K. Shultis and D. S. McGregor, IEEE Trans. Nucl. Sci. 53, 1659 (2006).

${ }^{6}$ R. J. Nikolic, C. L. Cheung, C. E. Reinhardt, and T. F. Wang, Proc. SPIE, 6013, 36 (2005).

${ }^{7}$ R. J. Nikolic, A. M. Conway, C. E. Reinhardt, R. T. Graff, T. F. Wang, N. Deo, and C. L. Cheung, Proceedings of the IEEE Nuclear Science Symposium, Honolulu (IEEE, New York, 2007), p. 1577.

${ }^{8}$ G. F. Knoll, Radiation Detection and Measurement, 3rd. ed. (Wiley, New York, 2000).

${ }^{9}$ A. M. Conway, R. J. Nikolic, and T. F. Wang, Proceedings of the International Semiconductor Device Research Conference, College Park, MD, 12-14 December 2007 (IEEE, New York, 2007), p. 589.

${ }^{10}$ N. Deo, J. R. Brewer, C. E. Reinhardt, R. J. Nikolic, and C. L. Cheung, J. Vac. Sci. Technol. B 26, 1309 (2008).

11“MCNP4C-Monte Carlo N-Particle Transport Code System, Los Alamos National Laboratory, (2000).

${ }^{12} \mathrm{~N}$. Tsoulfanidis, Measurement and Detection of Radiation, 2nd ed. (Taylor \& Francis, Washington, 1995).
} 